\title{
Abordagem da Hemostase e Anticoagulação no Contexto da Infeção Por SARS-CoV-2
}

\author{
Haemostasis Monitoring and Management of Anticoagulant Therapy in \\ SARS-COV-2 Infection
}

Teresa Seara Sevivas' (https://orcid.org/0000-0001-9770-1425), André Caiado² (https://orcid.org/0000-0002-9734-5800), Anabela Rodrigues ${ }^{3}$ (https://orcid.org/0000-0001-9981-030X), António Robalo Nunes ${ }^{4}$ (https://orcid.org/0000-00019384-7080)

Palavras-chave: Anticoagulantes; Coagulação Sanguínea; COVID-19; Infecções por Coronavírus; Tromboembolia Venosa.

Keywords: Anticoagulants; Blood Coagulation; Coronavirus Infections; COVID-19; Venous Thromboembolism.

\section{Introdução}

A doença associada à infeção por SARS-CoV-2 (COVID19), tornou-se numa pandemia, com o primeiro caso em Portugal declarado a 2/3/2020.

A síndrome respiratória aguda, evolui em muitos doentes para choque séptico, acidose metabólica e coagulopatia intravascular disseminada (CID). Estes doentes apresentam frequentemente alterações da coagulação, algumas associadas a pior prognóstico. ${ }^{1}$

O risco de complicações trombóticas, coagulopatia e CID nos doentes com COVID-19 recomenda a emissão de orientações relativas à monitorização da hemostase e terapêutica anticoagulante que pode beneficiar estes doentes.

O obejctivo foi sumarizar a literatura actual (com evidência clínica e pareceres das sociedades científicas) sobre diagnóstico e tratamento das alterações da hemostase na infecção por SARS-CoV-2.

\section{Material e Métodos}

Foi efectuada pesquisa bibliográfica na PubMed e Medline, usando as palavras-chave: "COVID-19», "SARSCoV infection», «thromboprophylaxis», «anticoagulation», «thrombosis» e "coagulopathy». A pesquisa bibliográfica e experiência clínica dos autores constitui a base deste manuscrito.

\footnotetext{
'Serviço de Sangue e Medicina Transfusional, Centro Hospitalar e Universitário de Coimbra, Coimbra, Portugal

${ }^{2}$ Serviço de Imunohemoterapia, Centro Hospitalar Universitário de Lisboa Central, Hospital de São José, Lisboa, Portugal

${ }^{3}$ Serviço de Imunohemoterapia, Centro Hospitalar de Lisboa Norte, Hospital Santa Maria, Lisboa, Portugal

${ }^{4}$ Serviço de Medicina Transfusional, Hospital das Forças ArmadasPólo de Lisboa, Lisboa, Portugal

https://revista.spmi.pt - DOI: 10.24950/A.Recentes/72/20/2/2020
}

\section{Princípios}

\section{A. REVISÃO DOS ESTÁDIOS DA COVID-19}

- Estádio I (fase precoce): a replicação viral que condiciona efeito citopático directo e ativação da resposta imune inata, associando-se a linfopenia e elevação dos D-dímeros e desidrogenase láctica (LDH)2 (Tabela 1);

- Estádio II (fase pulmonar): a ativação da resposta imune adaptativa resulta em redução da virémia, iniciando a cascata inflamatória capaz de causar lesão tecidual, associada a agravamento da linfopenia e elevação moderada da proteína $\mathrm{C}$ reactiva $(\mathrm{PCR})$ e transaminases ${ }^{2}$;

- Estádio III (fase hiperinflamatória): insuficiência multiorgânica fulminante com frequente agravamento do envolvimento pulmonar, que decorre de reposta imune desregulada condicionando uma síndrome de tempestade de citocinas $^{3}$ reminiscente da linfo-histiocitose hemofagocítica secundária. $^{2}$

\section{B. HIPERCOAGULABILIDADE}

Existe outro mecanismo importante associado ao efeito citopático do vírus sobre o endotélio e à resposta inflamatória - estado de hipercoagulabilidade. .,3-5 $^{13}$

A disfunção da célula endotelial induzida por infeção, resulta em excesso de geração de trombina e diminuição da fibrinólise, o que induz um estado de hipercoagulabilidade nos doentes com infeção ${ }^{3,6}$ como o COVID-19.

A hipóxia nos doentes com COVID-19 grave, pode estimular a trombose através da hiperviscosidade sanguínea e activação de vias de sinalização dependentes de fatores de transcrição hipóxia-dependentes. ${ }^{3}$ Foi descrita a ocorrência de microtromboses e oclusão de pequenos vasos pulmonares em doentes críticos com COVID-19. ${ }^{3}$ Estas contribuem para o agravamento da hipóxia (por alteração da relação ventilação/perfusão) e, mais tardiamente, associam-se a formas de CID com repercussão sistémica. ${ }^{2}$

A imobilização aumenta também o risco de tromboembolismo venoso (TEV) nestes doentes.

Assim, a introdução precoce de terapia anticoagulante com heparina de baixo peso molecular (HBPM) em doentes com COVID-19 é indicada por várias sociedades científicas, ${ }^{3,4}$ existindo actualmente algoritmos de actuação específicos 
Tabela 1: Scores SIC e DIC da ISTH. 3,8,9

\begin{tabular}{|c|c|c|c|c|c|}
\hline \multicolumn{3}{|c|}{ ISTH SIC Scoring System ${ }^{3}$} & \multicolumn{3}{|c|}{ ISTH DIC Scoring System ${ }^{8,9}$} \\
\hline Item & Score & Variação & Item & Resultado & Score \\
\hline $\begin{array}{c}\text { № Plaquetas } \\
\left(\times 10^{9} / \mathrm{L}\right)\end{array}$ & $\begin{array}{l}1 \\
2\end{array}$ & $\begin{array}{c}100-150 \\
<100\end{array}$ & $\begin{array}{c}\text { No Plaquetas } \\
\left(\times 10^{9} / \mathrm{L}\right)\end{array}$ & $\begin{array}{l}>100 \\
<100 \\
<50\end{array}$ & $\begin{array}{l}0 \\
1 \\
2\end{array}$ \\
\hline TP - INR & $\begin{array}{l}1 \\
2\end{array}$ & $\begin{array}{c}1,2-1,4 \\
>1,4\end{array}$ & TP & $\begin{array}{c}\uparrow<3 s \\
\uparrow>3 s-<6 s \\
\uparrow>6 s\end{array}$ & $\begin{array}{l}0 \\
1 \\
2\end{array}$ \\
\hline SOFA Score & $\begin{array}{l}1 \\
2\end{array}$ & $\begin{array}{c}1 \\
\geq 2\end{array}$ & $\begin{array}{c}\text { Fibrinogénio } \\
(g / L)\end{array}$ & $\begin{array}{l}>1,0 \\
<1,0\end{array}$ & $\begin{array}{l}0 \\
1\end{array}$ \\
\hline SIC Score & $\geq 4$ & & PDF/ & Sem aumento & 0 \\
\hline \multirow{3}{*}{\multicolumn{3}{|c|}{$\begin{array}{c}\text { Considerar SIC se: } \\
\text { SIC Score } \geq 4 \\
+ \\
\text { No Plaquetas }=2 \\
+ \\
\text { TP }- \text { INR }=2\end{array}$}} & D-Dímeros & $\begin{array}{c}\uparrow \text { Moderado } \\
\text { Grande } \uparrow\end{array}$ & $\begin{array}{c}2(250-5,000) \\
3(>5,000)\end{array}$ \\
\hline & & & $\begin{array}{l}\text { DIC Score } \\
\geq 4^{8}-5^{9}\end{array}$ & \multicolumn{2}{|c|}{ Compatível com CID } \\
\hline & & & $\begin{array}{l}\text { DIC Score } \\
\geq 4^{8}-5^{9}\end{array}$ & \multicolumn{2}{|c|}{$\begin{array}{l}\text { Sugestão de CID não evidente. } \\
\text { Recomenda-se repetir em 24-72h }\end{array}$} \\
\hline
\end{tabular}

$\uparrow$ Aumento; INR, International Normalized Ratio; CID, Coagulaccão intravascular disseminada; DIC, Disseminated instravascular coagulation ISTH, International Society on Thrombosis and Haemostasis; PDF, Produtos de degradação da fibrina; SIC, Sepsis-induced coagulopathy; SOFA, Sequential organ failure assessment; TP, Tempo de protrombina.

adoptados em vários hospitais e unidades de cuidados intensivos 2,3 (Tabela 2).

\section{ALTERAÇÕES HEMOSTÁTICAS}

Como a disfunção de órgão nas etapas iniciais da COVID -19 é principalmente limitada ao pulmão e o vírus é o principal patógeno, as alterações de coagulação provocadas neste contexto podem não ser idênticas às verificadas na sépsis em geral. ${ }^{4}$

A trombocitopenia é frequentemente considerada indicador de mortalidade por sépsis. não sendo este o caso em muitos doentes com COVID-19. ${ }^{4}$ Tal pode dever-se ao aumento reactivo da trombopoietina após inflamação pulmonar, ${ }^{3}$ pelo que a contagem plaquetária parece não ser um marcador sensível para a coagulopatia associada à COVID-19.3,4

Existe evidência de que os mais idosos e aqueles com comorbilidades (ambos os grupos tendem a apresentar Ddímeros mais elevados) têm maior probabilidade de morte pela COVID-19.,4 Um valor mais elevado de D-dímeros e maior prolongamento do tempo de protrombina (TP) na admissão hospitalar, foram associados a pior prognóstico em doentes com COVID-19 e positivamente correlacionados com a taxa de mortalidade aos 28 dias. , $^{13,7}$

$\mathrm{Na}$ altura do diagnóstico de COVID-19, D-dímeros elevados são considerados factor de mau prognóstico, enquanto a trombocitopenia se mostrou um indicador de prognóstico mais inconsistente. ${ }^{3}$

Alguns autores ${ }^{1,3}$ advogam que doentes em que se verifique aumento significativo de D-dímeros (3-4 vezes o valor
normal-VN), deve ser considerado internamento, mesmo na ausência de outros sintomas de gravidade, uma vez que isso significa aumento da geração de trombina, ${ }^{1,3} \mathrm{com}$ pior prognóstico a curto prazo.

\section{SCORE SIC}

A diminuição do número de plaquetas e prolongamento do TP correlacionam-se com aumento da mortalidade na sépsis. Mas uma vez que a hipofibrinogenémia é incomum na sépsis, a International Society on Thrombosis and Hemostasis (ISTH) propôs uma nova categoria de coagulopatia na sépsis, identificando uma fase mais precoce, denominada sepsis induced coagulopathy $(\mathrm{SIC})^{3}$ (Tabela 1). Substitui-se assim inicialmente o score de CID nestes doentes pelo score SIC, que permite orientar precocemente a terapia anticoagulante, sempre que estes apresentem score $\mathrm{SIC} \geq 4^{3}$ (Tabela 2).

\section{E. ANTICOAGULAÇÃO}

O tratamento com HBPM parece associar-se a melhor prognóstico em doentes com COVID-19 e coagulopatia. ${ }^{1-4,8,9}$ Já foi possível analisar retrospectivamente séries robustas de casos de infeção grave na China e avaliar diferenças entre os doentes do início da pandemia que não beneficiaram de anticoagulação, e aqueles diagnosticados mais tardiamente no surto, que foram anticoagulados. ${ }^{1,3}$ Verificou-se no estudo de Tang $\mathrm{N}$ et al, (449 doentes), que, em doentes COVID-19 com SIC score ${ }^{3,4}(p=0,029)$ ou D-dímeros $>3 \mathrm{ug} / \mathrm{mL}$ (>6 vezes o VN; $p=0,017$ ) a taxa de mortalidade foi significativamente menor em doentes submetidos a anticoagulação com HBPM. ${ }^{2}$ 
Tabela 2: Protocolo de tromboprofilaxia na COVID-194,8,9,10. Adaptado Hospital LA PAZ

- Laboratório: D-Dímeros, TP, Hemograma, Fibrinogénio, TTPa ${ }^{4}$

- Avaliar sempre risco de hemorragia e contraindicação para uso de HBPM ${ }^{8}$

- Anticoagulação oral prévia (AVK/ACOD): suspender e mudar para HBPM (sc) em doses terapêuticas ${ }^{8,9}$

- Antiagregação Plaquetária: MONOTERAPIA $\rightarrow$ Aspirina; DUPLA $\rightarrow$ Aspirina+HBPM (dose anticoagulante) ou Prasugrel ${ }^{9}$

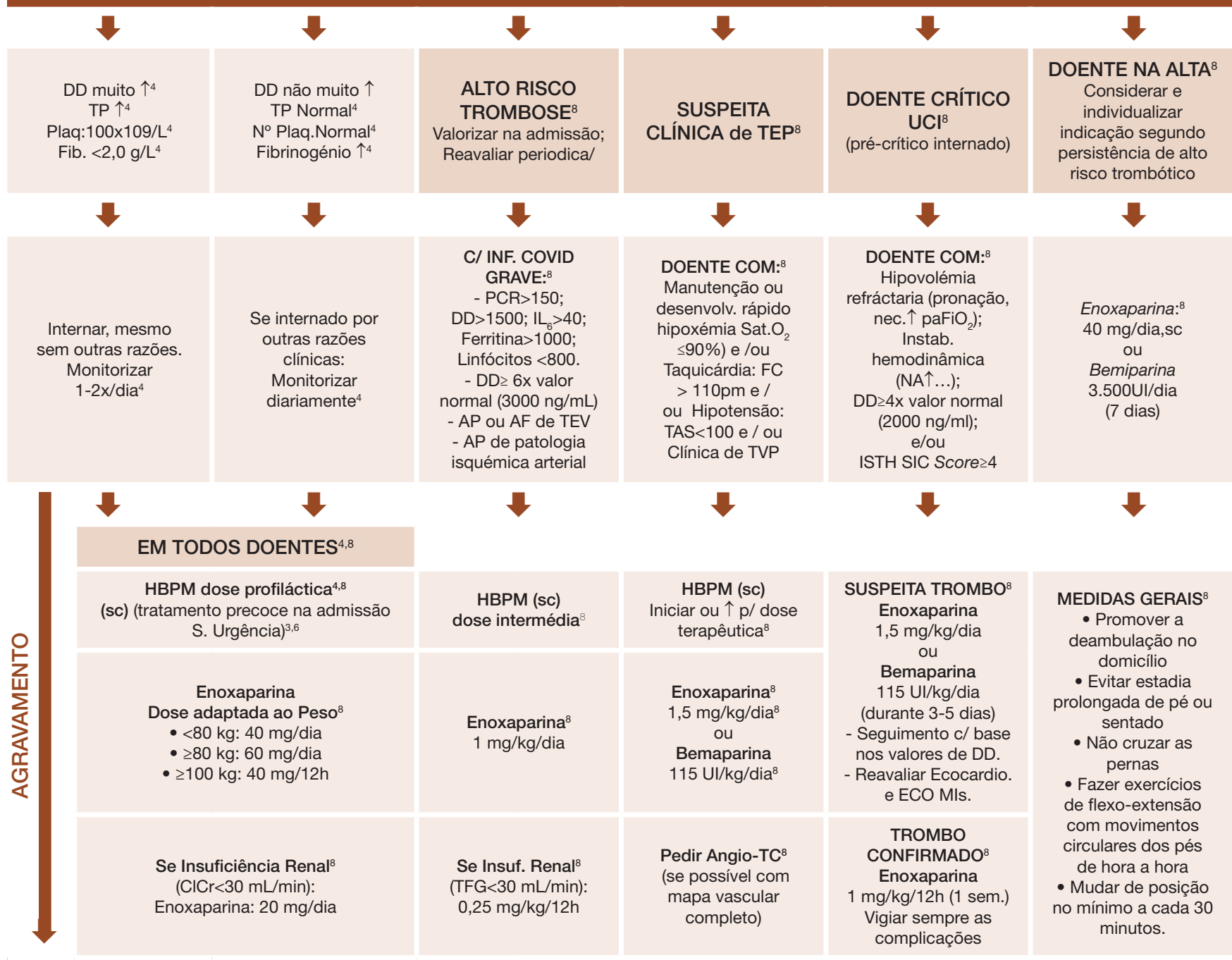

- Terap. Transfusional segundo protocolo local 4,8

- Eventual tratamento experimental ${ }^{4}$

Se Hemorragia Grave - Alvos Terapêuticos 4,10

- Hb: 7-9 g/dl; Plaq. $>50 \times 10 \% / L$; Fib. $>2,0$ g/L; TP/TTPa $<1,5 x$ VN

- $\mathrm{Ca}^{2+i}>1,2 \mathrm{mmol} / \mathrm{L} ; \mathrm{pH}>7,2 ;$ Lactato<4 mmol/L; Défice bases<-3; Sat. $\mathrm{O}_{2}>92 \%$

$\uparrow$ Aumento; ACOD, Anticoagulantes orais directos; AF, Antecedentes familiares; AP, Antecedentes pessoais; AVK, Antagonistas da vitamina K; bpm, batimentos cardíacos por minuto; c/, com; Ca2+i, Cálcio ionizado; card., cardíaca; CICr, Clearance da creatinina; d, dia; DD, D-Dímeros; desenvolv., desenvolvimento; dl, decilitro; ECO Mls, Ecografia dos membros inferiores; Ecocardio, Ecocardiograma; FC, Frequência cardíaca; Fib.,Fibrinogénio; h, hora; Hb, Hemoglobina; HBPM, Heparina de baixo peso molecular; IL, Interleucina; instab., instabilidade; Insuf., Insuficiência; ISTH,International Society on Thrombosis and Haemostasis; kg, quilogramas; L, litros; mg, miligramas; mL, mililitros; NA, noradrenalina; nec., necessidade; neuro, neurológica; ng, nanogramas; p/, para; PCR, Proteína C-reactiva; perif., periférica; Perif., Periférico; Plaq.,Plaquetas; S., Serviço de; Sat.O2, Saturação periférica de oxigénio; sc, subcutânea; sem., semana; SIC, Sepsis-induced Coagulopathy; TAS, tensão arterial sistólica; TC, Tomografia computorizada; TEP, Tromboembolismo pulmonar; Terap., Terapêutica; TEV, Tromboembolismo venoso; TFG, Taxa de filtração glomerular; TP, Tempo de protrombina; TTPa, Tempo de tromboplastina activada; TVP, trombose venosa profunda; UCI, Unidade de cuidados intensivos; UI, Unidades internacionais; $\mathrm{VN}$, valor normal; $\mathrm{x}$, vezes.

O tratamento com heparina associou-se a menor mortalidade em doentes com score $\mathrm{SIC} \geq 4$, mas não naqueles com score $\mathrm{SIC}<4 .{ }^{1,3}$ Perante valor de D-dímeros > 3,0 ug/ $\mathrm{mL}$ (6 vezes o VN) verifica-se uma redução significativa (aproximadamente 20\%) na mortalidade quando os doentes são tratados com HBPM. ${ }^{3,4}$

A HBPM foi o anticoagulante mais utilizado nessas avaliações retrospectivas para prevenir CID e TEV em doentes com 
COVID-19, devido ao seu efeito anti-inflamatório. ${ }^{3}$ Foi usada dose profilática de HBPM e as complicações hemorrágicas foram raras e ligeiras.

\section{F. ANTICOAGULAÇÃO COM HBPM}

A inibição da geração de trombina parece ter benefício na redução da mortalidade associada à COVID-19. ${ }^{4}$ O único tratamento amplamente disponível é a anticoagulação profilática com HBPM, que actualmente deve ser considerada em TODOS os pacientes (incluindo não críticos) que requerem internamento, na ausência de contraindicações (hemorragia activa e contagem de plaquetas inferior a $\left.25 \times 10^{9} / \mathrm{L}\right){ }^{4}$ Aconselha-se monitorização adequada perante compromisso grave da função renal. ${ }^{4}$ Valores anormais de TP e tempo tromboplastina parcial activado (TTPa) não são contraindicação à terapêutica profiláctica com HBPM. ${ }^{4}$

A HBPM também protege doentes críticos contra TEV e as propriedades anti-inflamatórias podem ser um benefício na COVID-19, em que as citocinas pró-inflamatórias estão elevadas. ${ }^{2,4}$

\section{Pontos importantes}

- Factores de pior prognóstico no doente infectado com SARS-CoV-2:

- $\quad$ elevação D-dímeros, ${ }^{8}$ especialmente se >3,0 ug/mL (6 vezes maior que $\mathrm{VN})^{1,3,4}$

- prolongamento TP,4 especialmente se >1,5 vezes em relação ao valor de referência ${ }^{3}$

-- score SIC elevado ${ }^{4}$, especialmente $\mathrm{se}^{3,4}$

- score CID elevado ${ }^{1,3,4,8,9}$

- O doente infectado com SARS-CoV-2 tem um estado de hipercoagulabilidade com microtromboses e oclusão de pequenos vasos pulmonares, que agravam a hipóxia (a qual agrava as microtromboses pulmonares) e, mais tardiamente, associam-se a formas de CID com repercussão sistémica. ${ }^{1-4,8.9}$ Adicionalmente, a imobilização aumenta o risco de TEV. ${ }^{4}$

- A ISTH propôs uma nova categoria que identifica numa fase mais precoce a coagulopatia na sépsis- SIC, cujo score deve ser aplicado a estes doentes, permitindo orientar precocemente a terapia anticoagulante. ${ }^{4}$

- O único tratamento amplamente disponível para inibir a geração de trombina é a HBPM (fundaparina ou bemiparina em alternativa se enoxaparina estiver contraindicada) $)^{4,8,9}$ que actualmente deve ser considerada em TODOS os doentes que requerem internamento por COVID-19, na ausência de contraindicações (hemorragia activa e contagem de plaquetas inferior a $25 \times 109 / \mathrm{L})$. $^{3,4}$ Deve efectuar-se monitorização adequada no caso de compromisso grave da função renal. ${ }^{4}$

- HBPM precoce diminui o risco de mortalidade aos 7 e 28 dias em $48 \%$ e $7 \%$ respectivamente. ${ }^{8}$

\section{Recomendações ${ }^{1-4,8,9}$}

\section{A. MONITORIZAÇÃO}

1. Rastreio da coagulação na admissão, como monitorização de doentes infectados com SARS-CoV-2, e no seguimento dos casos mais graves;

2. O rastreio deve ser precoce e incluir D-dímeros, TP e contagem de plaquetas, por ordem decrescente de importância, em TODOS os doentes com COVID-19;

3. Avaliar score SIC (precoce) e CID (mais tardio) (Tabela 1);

4. Pode ser útil dosear o fibrinogénio neste cenário, conforme recomendado pela ISTH4;

5. Considerar: monitorização de estados de hiperfibrinólise e hiperfibrinólise associada ou agravada por ECMO. Neste caso, dosear FXIII e antitrombina;

6. Monitorização de estados de hipercoagulabilidade, por testes viscoelásticos, quando disponíveis ${ }^{6}$;

7. Considerar doseamento da actividade anti-FXa, sobretudo nos doentes com baixa taxa de filtração glomerular (TFG<50 mL/min).

\section{B. TERAPÊUTICA}

1. Considerar HBPM profiláctica em TODOS os doentes (incluindo não críticos) internados por COVID-19, na ausência de contraindicações (hemorragia activa e contagem de plaquetas inferior a 25×109/L).3,4 Efectuar monitorização adequada no caso de insuficiência renal grave. ${ }^{4}$

2. Valores anormais de TP e TTPa não são contraindicação à terapêutica profiláctica com HBPM. ${ }^{4}$

Responsabilidades Éticas

Conflitos de Interesse: Os autores declaram a inexistência de conflitos de interesse na realização do presente trabalho.

Fontes de Financiamento: Não existiram fontes externas de financiamento para a realização deste artigo

Confidencialidade dos Dados: Os autores declaram ter seguido os protocolos da sua instituição acerca da publicação dos dados de doentes.

Proteção de Pessoas e Animais: Os autores declaram que os procedimentos seguidos estavam de acordo com os regulamentos estabelecidos pelos responsáveis da Comissão de Investigação Clínica e Ética e de acordo com a Declaração de Helsínquia da Associação Médica Mundial. Proveniência e Revisão por Pares: Não comissionado; revisão externa por pares.

\section{Ethical Disclosures}

Conflicts of interest: The authors have no conflicts of interest to declare. Financing Support: This work has not received any contribution, grant or scholarship.

Confidentiality of Data: The authors declare that they have followed the protocols of their work center on the publication of data from patients.

Protection of Human and Animal Subjects: The authors declare that the procedures followed were in accordance with the regulations of the 
relevant clinical research ethics committee and with those of the Code of Ethics of the World Medical Association (Declaration of Helsinki).

Provenance and Peer Review: Not commissioned; externally peer reviewed

(C) Autor (es) (ou seu (s) empregador (es)) 2019. Reutilização permitida de acordo com CC BY-NC. Nenhuma reutilização comercial.

(C) Author(s) (or their employer(s)) 2019. Re-use permitted under CC BY-

NC. No commercial re-use.

\section{Correspondence / Correspondência:}

Teresa Seara Sevivas - teresasevivas@hotmail.com

Serviço de Sangue e Medicina Transfusional, Centro Hospitalar e Universitário de Coimbra, Coimbra, Portugal

Praceta Pro. Mota Pinto, 3000-075 Coimbra

Received / Recebido: 22/04/2020

Accepted / Aceite: 06/05/2020

Publicado / Published: 27 de Junho de 2020

\section{REFERÊNCIAS}

1. Tang N, Li D, Wang X, Sun Z. Abnormal coagulation parameters are associated with poor prognosis in patients with novel coronavirus pneumonia. J Thromb Haemost. 2020 (in press). doi: 10.1111/jth.14768.

2. Mendes J, Mergulhão P, Froes F, Paiva JA, Gouveia J. Recomendações da Sociedade Portuguesa de Cuidados Intensivos e Grupo Infeção e Sépsis para a abordagem do COVID-19 em Medicina Intensiva. Rev Bras Terap Intensiva 2020 (in press). doi.org/10.5935/0103-507X.

3. Tang N, Bai H, Chen X, Gong J, Li D, Sun Z. Anticoagulant treatment is associated with decreased mortality in severe coronavirus disease 2019 patients with coagulopathy. J Thromb Haemost. 2020 (in press). doi: 10.1111/ JTH.14817.

4. Tachil J, Tang N, Gando S, Falanga A, Cattaneo M, Levi M, Clark C, Iba T. ISTH interim guidance on recognition and management of coagulopathy in COVID-19. J Thromb Haemost. 2020 (in press). doi: 10.1111/JTH.14810.

5. Gralinski LE, Bankhead A, Jeng $S$ et al. Mechanisms of severe acute respiratory syndrome coronavirus-induced acute lung injury. mBio. 2013; 4: e00271-e313.

6. Wilson AJ, Martin DS, Maddox V, Rattenbury S, Bland D, Bhagani S, et al. Thromboelastography in the Management of Coagulopathy Associated with Ebola Virus Disease. Clin Infect Dis. 2016;62:610-2.

7. Beeching NJ, Fletcher TE, Fowler R. BMJ Best Practice COVID-19. [accessed May 2020] Available from: http://bestpractice.bmj.com.

8. Webinar SETH (Sociedad Española de Trombosis y Hemostais). Coagulación, Anticoagulación y COVID-19: lo que te interesa conocer. MODERADOR: Dr. José A. Páramo Fernández, Serviço hematologia CUN, Pamplona. Oradores: CORONAVIRUS Y COAGULOPATÍA-Dr.a Olga Gavín Sebastián, INTERPRETACIÓN LABORATORIO DE HEMOSTASIA-Dr. Joan Carles Reverter Calatayud, DOCUMENTO SETH SOBRE ANTICOAGULANES Y COVID-19-Dr. Pere Domènech Santasusana, PREVENCIÓN Y TRATAMIENTO DE LA COAGULOPATÍA ASOCIADA A COVID-19-Dr. Víctor Jiménez Yuste. Emitido em 06.04.2020 às 13h. https://www.covid-19.seth.es/coagulacion-anticoagulacion-y-covid-19-lo-que-te-interesa-conocer/

9. Llau J.V., Ferrandis R, Sierra P, Hidalgo F, Cassinello C, Gómez-Luque A, Quintana M, Amezaga R, Gero M, Serrano A, Marcos P. Recomendaciones de consenso SEDAR-SEMICYUC sobre el manejo de las alteraciones de la hemostasia en los pacientes graves con infección por COVID-19. SEDAR-SEMICYUC consensus recommendations on the management of haemostasis disorders in severely ill patients with COVID-19 infection. Rev.Esp. Anesthesiol. Reanim. 2020: 23:1-16. doi.org/10.1016/j.redar.2020.05.007

10. Carvalho M, Rodrigues A, Gomes M, Carrilho A, Robalo Nunes A, Orfão $\mathrm{R}$,et al. Interventional Algorithms for the controlo of coagulopathic bleeding in surgical, trauma and pospartum settings. Clin Applied Thrombosis/Hemostasis. 2014:1-17 